\title{
Numerical methods for calculation of compound rods
}

\author{
Bulat Kuzhin* \\ Moscow State University of Civil Engineering, Yaroslavskoe shosse, 26, Moscow, 129337, Russia
}

\begin{abstract}
Presented calculation of the compound rod for free oscillations and test tasks of calculation of monolithic rods, which in fact began testing the above methods, which the authors considered necessary before using these methods on more complex - composite rods, the results of the applied methods are compared. The purpose of this work - the calculation of the composite rods on the dynamic effects of the method of successive approximations and finite element method, comparing the results; determining the limits of rational use of successive approximations method (SAM). The method of successive approximations (SAM) is used as a resolving method of differential equations. The application of this method to the calculation of structures has a number of advantages: the ability to consider a simplified design scheme of the beam with a distributed load (instead of a system of concentrated forces); the ability to abandon the direct simulation of the design scheme in the design and computer complex (the time of which depends on the number of connections of the composite beam); gives the result of high accuracy at low time costs.
\end{abstract}

\section{Introduction}

In reinforced concrete constructions to the scheme of compound beam can be converted supporting structures of multistory buildings (Fig. 1). Crossbars and jumpers plays the same role as a bar in a stele column here. Calculation of structures for dynamic effects is the most difficult task of structural mechanics. This problem is described by a system of differential equations of motion, for solving which it is necessary to involve a diverse arsenal of mathematical methods. The dynamic calculation uses well-developed methods of statics, but essentially generalizes them with the help of the D'Alembert's principle, introducing a new variable into the equation - time, i.e. takes into account the occurrence of inertial forces $[1,2,3,4]$. The purpose of the dynamic calculation of structures is as follows: calculation of natural oscillations and determination of the main dynamic characteristics; calculation of forced oscillations and determination of the amplitude of dynamic movements and verification of physiological and technological requirements to limit the level of oscillations; determination of dynamic loads and their classification by frequency and dynamism; determination of the amplitude of internal forces in structures and the calculation of strength and endurance. The following methods are used in the calculation of

\footnotetext{
* Corresponding author: kuzhinbf@gmail.com
} 
test problems: the finite-difference method (FDM), the method of successive approximations (SAM), the analytical methods of Korenev and Rzhanitsyn, the finite element method (FEM) $[5,6,7,8]$.
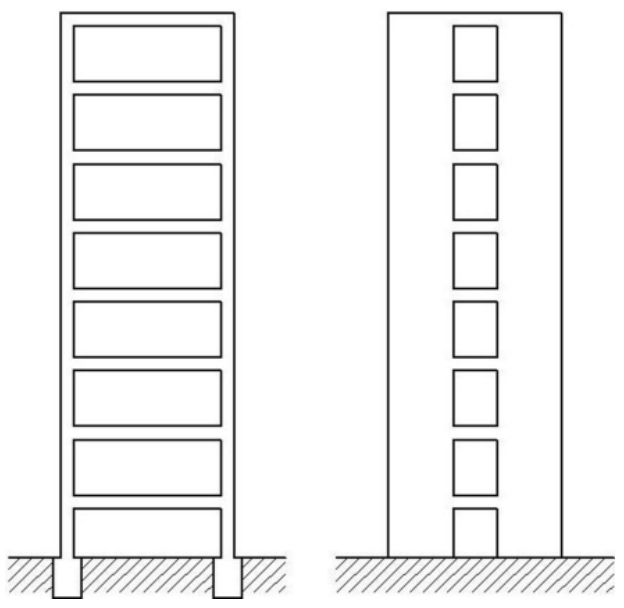

Fig. 1. Multistory building.

\section{Test tasks}

\subsection{The equation for the natural oscillations. SAM method}

Compare the frequency of the first form of vibrations obtained SAM, FDM, according to Korenev and FEM for beams with a cross section dimensions $0.4 \mathrm{~m}$ x $0.6 \mathrm{~m}$ (Fig. 2).
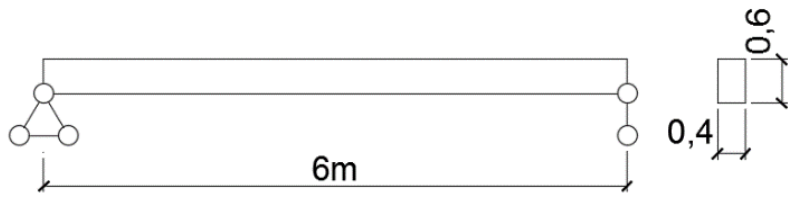

Fig. 2. A monolithic beam.

The equation for oscillation $[9,10,11,12]$ :

$$
\frac{\partial^{2}}{\partial x^{2}}\left[\operatorname{EI}(x) \frac{\partial^{2} \mathrm{~W}}{\partial \mathrm{x}^{2}}\right]=\mu(\mathrm{x}) \omega^{2} \mathrm{~W}(\mathrm{x}),(1.1 .1)
$$

Lower the differential equation:

$$
\left\{\begin{array}{c}
\mathrm{EI}(\mathrm{x}) \frac{\partial^{2} \mathrm{~W}}{\partial \mathrm{x}^{2}}=-\mathrm{M},(1.1 .2 \mathrm{a}) \\
\frac{\partial^{2} \mathrm{M}}{\partial \mathrm{x}^{2}}=\mu(\mathrm{x}) \omega^{2} \mathrm{~W}(\mathrm{x})(1.1 .26)
\end{array}\right.
$$


And then convert into dimensionless form:

$$
\left\{\begin{array}{c}
\frac{\partial^{2} \mathrm{w}}{\partial \xi^{2}}=-\mathrm{gm},(1.1 .3 \mathrm{a}) \\
\frac{\partial^{2} \mathrm{~m}}{\partial \xi^{2}}=-\gamma \rho^{2} \mathrm{w}(1.1 .36)
\end{array}\right.
$$

Further, using the SAM method, differential equations are approximated on a uniform grid with step h (Fig. 3) the following difference equations $[13,14,15,16]$ :

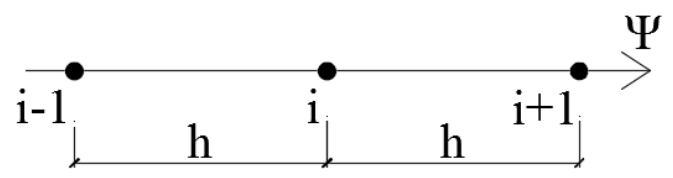

Fig. 3. The scheme of the uniform mesh with the step h.

$$
\begin{gathered}
{ }^{\pi} W_{i-1}-2{ }^{\pi} W_{i}+{ }^{\pi} W_{i+1}=-\frac{h^{2}}{12}\left({ }^{\pi} m_{i-1}+10{ }^{\pi} m_{i}+{ }^{\pi} m_{i+1}\right),(1.1 .4) \\
{ }^{\pi} m_{i-1}-2{ }^{\pi} m_{i}+{ }^{\pi} m_{i+1}=-\frac{h^{2}}{12} \rho^{2}\left({ }^{\pi} W_{i-1}+10{ }^{\pi} W_{i}+{ }^{\pi} W_{i+1}\right),(1.1 .5)
\end{gathered}
$$

We obtain a system of equations by (1.1.4) and (1.1.5):

$$
\left\{\begin{array}{c}
-2 \mathrm{~W}_{1}=-(1 / 2)^{2} \frac{10}{12} \mathrm{~m}_{1} \\
-2 \mathrm{~m}_{1}=-(1 / 2)^{2} \frac{10}{12} \rho^{2} \mathrm{~W}_{1}
\end{array}\right.
$$

Solving the system, we obtain the desired dimensionless value for the frequency of the first form of oscillations:

$$
\rho=9,6
$$

Further transform from the dimensionless value to the dimensional form, having previously taken:

$$
\begin{gathered}
\mathrm{b}=0,4 ; \mathrm{h}=0,6 ; \mathrm{E}=3 \cdot \frac{10^{7} \mathrm{kN}}{\mathrm{m}^{2}} ; \mu=0,2 ; \rho=2200 \frac{\mathrm{kg}}{\mathrm{m}^{3}} \\
\omega=\sqrt{\frac{\rho^{2} \mathrm{EI}_{0}}{\mu_{0} 1^{4}}}=\sqrt{\frac{92,16 \cdot 3 \mathrm{e} 10 \cdot 0,0072}{528 \cdot 6^{4}}}=170,56\left(\frac{\mathrm{rad}}{\mathrm{s}}\right) \times \frac{1}{2 \pi}=27,16(\mathrm{~Hz}) ;
\end{gathered}
$$




\subsection{The calculation method of FDM}

For regular points:

$$
\begin{gathered}
{ }^{\pi} \mathrm{W}_{\mathrm{i}-1}-2{ }^{\pi} \mathrm{W}_{\mathrm{i}}+{ }^{\pi} \mathrm{W}_{\mathrm{i}+1}+\Delta \mathrm{W}_{\mathrm{i}}+\mathrm{h} \Delta \mathrm{W}_{\mathrm{i}}{ }^{\prime}==-\mathrm{h}^{2}\left({ }^{\pi} \mathrm{g}_{\mathrm{i}}-\frac{\Delta \mathrm{g}_{\mathrm{i}}}{2}\right){ }^{\pi} \mathrm{m}_{\mathrm{i}}+\frac{\mathrm{h}^{2}}{2}{ }^{ } \mathrm{g}_{\mathrm{i}} \Delta \mathrm{m}_{\mathrm{i}},(1.2 .1) \\
{ }^{ } \mathrm{m}_{\mathrm{i}-1}-2{ }^{\pi} \mathrm{m}_{\mathrm{i}}+{ }^{\Pi} \mathrm{m}_{\mathrm{i}+1}+\Delta \mathrm{m}_{\mathrm{i}}+\mathrm{h} \Delta \mathrm{m}_{\mathrm{i}}{ }^{\prime}==-\mathrm{h}^{2} \rho^{2}\left({ }^{\pi} \gamma_{\mathrm{i}}-\frac{\Delta \gamma_{\mathrm{i}}}{2}\right){ }^{\pi} \mathrm{W}_{\mathrm{i}}+\frac{\mathrm{h}^{2}}{2} \rho^{2}{ }^{ }{ }_{\mathrm{i}} \Delta \mathrm{W}_{\mathrm{i}},(1.2 .2)
\end{gathered}
$$

For boundary points:

$$
\begin{gathered}
-\mathrm{h}{ }^{ } \mathrm{W}_{\mathrm{i}}{ }^{\prime}-{ }^{ } \mathrm{W}_{\mathrm{i}}+{ }^{ } \mathrm{W}_{\mathrm{i}+1}=-\frac{\mathrm{h}^{2}}{2}{ }^{ } \mathrm{g}_{\mathrm{i}}{ }^{ } \mathrm{m}_{\mathrm{i}},(1.2 .3) \\
-\mathrm{h}{ }^{ } \mathrm{m}_{\mathrm{i}}{ }^{\prime}-{ }^{\mathrm{\Pi}} \mathrm{m}_{\mathrm{i}}+{ }^{ } \mathrm{m}_{\mathrm{i}+1}=-\frac{\mathrm{h}^{2}}{2} \rho^{2}{ }^{ } \gamma_{\mathrm{i}}{ }^{ } \mathrm{W}_{\mathrm{i}},(1.2 .4)
\end{gathered}
$$

We obtain a system of equations by (1.2.5) and (1.2.6):

$$
\left\{\begin{array}{l}
-2 \mathrm{~W}_{1}=-(1 / 2)^{2} \mathrm{~m}_{1} \\
-2 \mathrm{~m}_{1}=-(1 / 2)^{2} \rho^{2} \mathrm{~W}_{1}
\end{array}\right.
$$

Solving the system, we obtain the desired dimensionless value for the frequency of the first form of oscillations:

$$
\rho=8
$$

Further from the dimensionless value to the dimensional form:

$$
\omega=\sqrt{\frac{\rho^{2} \mathrm{EI}_{0}}{\mu_{0} \mathrm{l}^{4}}}=\sqrt{\frac{64 \cdot 3 \mathrm{e} 10 \cdot 0,0072}{528 \cdot 6^{4}}}=142,13\left(\frac{\mathrm{rad}}{\mathrm{s}}\right) \times \frac{1}{2 \pi}=22,63(\mathrm{~Hz}) ;
$$

Determine the natural frequencies from "Handbook on dynamics of structures" edited by B.

G. Korenev, 1972 [17]:

For the first frequency $\alpha^{2}=9,869$ :

$$
\theta_{\mathrm{s}}=\lambda_{\mathrm{s}}^{2} \sqrt{\frac{\mathrm{EI}}{\mathrm{m}}}, \text { где } \lambda=\frac{\alpha}{\mathrm{l}}
$$

$$
\begin{gathered}
\lambda^{2}=\frac{9,869}{6^{2}}=0,274139 \\
\theta_{1}=0,274139 \sqrt{\frac{3 \cdot 10^{10} \cdot 0,0072}{528}}=175,34 \frac{\mathrm{rad}}{\mathrm{s}}=27,92 \mathrm{~Hz}
\end{gathered}
$$

For the second frequency $\alpha_{2}^{2}=39,478$ :

$$
\begin{gathered}
\lambda_{2}^{2}=\frac{39,478}{6^{2}} \\
\theta_{2}=1,0966 \sqrt{\frac{3 \cdot 10^{10} \cdot 0,0072}{528}}=701,39 \frac{\mathrm{rad}}{\mathrm{s}}=111,69 \mathrm{~Hz}
\end{gathered}
$$

\subsection{The calculation method of FEM}

Fig. 4. FE model.

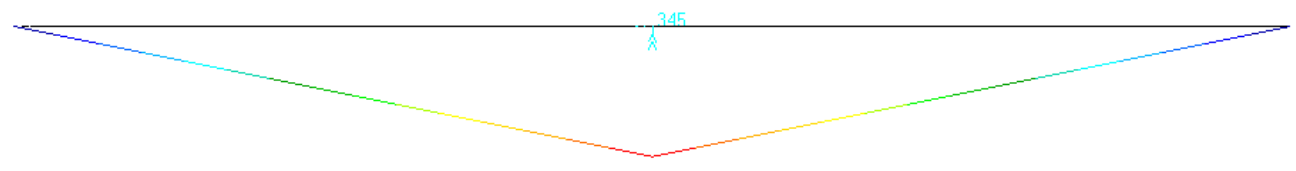

Fig. 5. First mode $(27,315 \mathrm{~Hz})$. 
Compare the results obtained by SAM, FDM, FEM and Korenev's handbook:

Table 1. Compare results.

\begin{tabular}{|c|c|}
\hline $\begin{array}{c}\text { Calculation } \\
\text { method }\end{array}$ & The value of the frequency of oscillation, $\mathrm{Hz}$ \\
\hline SAM & 27,16 \\
\hline FDM & 22,63 \\
\hline FEM & 27,315 \\
\hline Korenev & 27,92 \\
\hline
\end{tabular}

\section{Calculation of compound rods}

\subsection{The calculation method of SAM [4]}

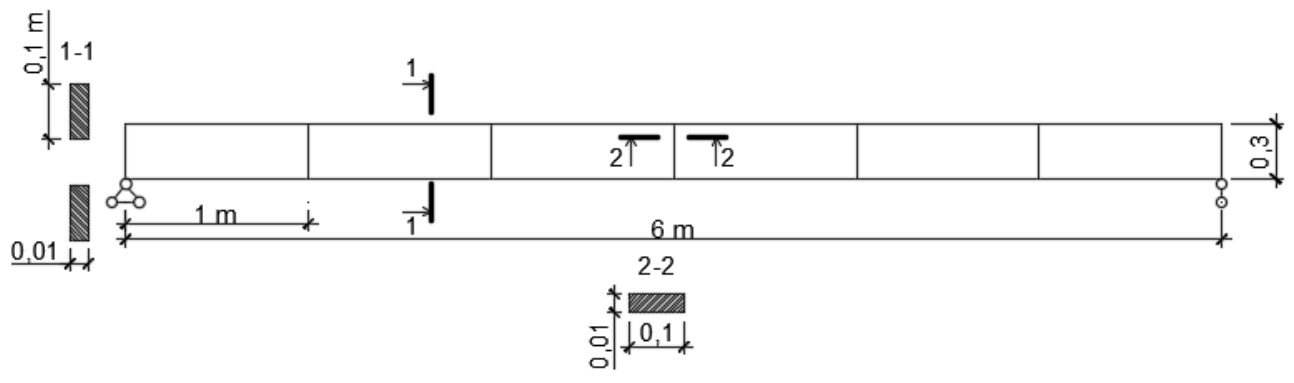

Fig. 6. The design scheme at a step of jumpers $h=1 \mathrm{~m}$

$$
\begin{gathered}
I=\frac{\mathrm{bh}^{3}}{12}=\frac{0,01 \cdot 0,1^{3}}{12}=8,(3) \cdot 10^{-7}\left(\mathrm{~m}^{4}\right) \\
\mathrm{E}=2,1 \cdot 10^{8}\left(\frac{\mathrm{KH}}{\mathrm{M}^{2}}\right) ; \rho=7,85\left(\frac{\mathrm{t}}{\mathrm{m}^{3}}\right) \\
\mathrm{F}=0,01 \cdot 0,1=10^{-3}\left(\mathrm{~m}^{2}\right)
\end{gathered}
$$

The stiffness of the seam $[18,19,20]$ :

$$
\begin{gathered}
\xi=\frac{24 \mathrm{EI}}{\mathrm{Bc}^{2}(2 \mathrm{c}+\mathrm{B})}=\frac{24 \cdot 2,1 \cdot 10^{11} \cdot 8,333 \cdot 10^{-7}}{1 \cdot 0,3^{2}(2 \cdot 0,3+1)}=\frac{420 \cdot 10^{4}}{0,144}=2916,(6) \cdot 10^{4}=2,9166 \cdot 10^{7} \\
\gamma=\frac{1}{\mathrm{E}}\left(\frac{2}{\mathrm{~F}}+\frac{\mathrm{c}^{2}}{2 \mathrm{I}}\right)=\frac{1}{2,1 \cdot 10^{11}}\left(\frac{2}{10^{-3}}+\frac{0,3^{2}}{2 \cdot 8,333 \cdot 10^{-7}}\right)=2,66666 \cdot 10^{-7} \\
\lambda^{2}=\gamma \xi=2,6666 \cdot 10^{-7} \cdot 2,9166 \cdot 10^{7}=7,77777 \\
\bar{\eta}=\lambda^{2} \cdot \mathrm{l}^{2}=7,77777 \cdot 36=279,9999 \approx 280
\end{gathered}
$$

Weight of rod (composite) per one linear meter: 


$$
\begin{gathered}
2 \mathrm{~F}=2 \cdot 10^{-3}\left(\mathrm{~m}^{2}\right) \\
2 \mathrm{~F} \rho=2 \cdot 10^{-3} \cdot 7850 \cdot \frac{\mathrm{kg}}{\mathrm{m}^{3}} \cdot 1 \mathrm{M}=15,7(\mathrm{~kg} / \mathrm{m})
\end{gathered}
$$

At step mesh $\mathrm{B}=1 \mathrm{~m}$ for every meter there is one stand

$$
\begin{gathered}
\mathrm{CF}=0,3 \cdot 10^{-3}=3 \cdot 10^{-4}\left(\mathrm{~m}^{3}\right) \\
\mathrm{CF} \rho=3 \cdot 10^{-4} \cdot 7850=2,355(\mathrm{~kg} / \mathrm{m})
\end{gathered}
$$

Weight per meter composite beams:

$$
15,7+2,355=18,055(\mathrm{~kg} / \mathrm{m})
$$

$$
\mathrm{z}=\frac{4 \mathrm{I}}{4 \mathrm{I}+\mathrm{Fc}^{2}}=\frac{4 \cdot 8,3333 \cdot 10^{-7}}{4 \cdot 8,3333 \cdot 10^{-7}+10^{-3} \cdot 0,3^{2}}=0,035714
$$

Check the solution:

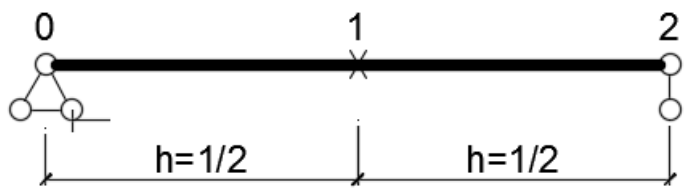

Fig. 7. The design scheme of SAM method at a step of jumpers $h=1 / 2$

$$
\begin{gathered}
\tau=\frac{1}{2} ; \Gamma . \mathrm{У} .: \varphi=\mathrm{W}=\bar{\omega}=0 \\
-2 \mathrm{~W}_{1}=\frac{1}{12} \cdot \frac{1}{2^{2}} \cdot 10 \varphi_{1} \\
-2 \varphi_{1}=\frac{1}{12} \cdot \frac{1}{2^{2}} \cdot 10 \bar{\omega}_{1} \\
-2 \bar{\omega}_{1}=-\frac{1}{12} \cdot \frac{1}{2^{2}}\left[\bar{\rho} \cdot 280 \cdot 0,035714 \cdot 10 \mathrm{~W}_{1}-\bar{\rho} 10 \varphi_{1}-280 \cdot 10 \bar{\omega}_{1}\right]
\end{gathered}
$$

The last equations can be rewritten:

$$
\begin{gathered}
-2\left(1+\frac{5}{12} \cdot \frac{1}{2^{2}} \cdot 280\right) \bar{\omega}_{1}=-\frac{1}{12} \cdot \frac{1}{2^{2}} \bar{\rho}\left[280 \cdot 0,035714 \cdot 10 \mathrm{~W}_{1}-10 \varphi_{1}\right] \\
-96 \mathrm{~W}_{1}=10 \varphi_{1} \\
-96 \varphi_{1}=10 \bar{\omega}_{1} \\
96\left(1+\frac{1400}{48}\right) \bar{\omega}_{1}=\bar{\rho}\left[100 \mathrm{~W}_{1}-10 \varphi_{1}\right] \\
\mathrm{W}_{1}=-\frac{10}{96} \varphi_{1} ; \varphi_{1}=-\frac{10}{96} \bar{\omega}_{1} \\
(96+2800) \bar{\omega}_{1}=\bar{\rho}\left[100 \mathrm{~W}_{1}-10 \varphi_{1}\right] \\
2896 \bar{\omega}_{1}=\bar{\rho}\left[-\frac{1000}{96} \varphi_{1}-\frac{960}{96} \varphi_{1}\right] \\
2896 \bar{\omega}_{1}=\bar{\rho}\left[-\frac{1960}{96} \varphi_{1}\right]
\end{gathered}
$$




$$
\begin{gathered}
2896 \bar{\omega}_{1}=\bar{\rho}\left[-\frac{1960}{96}\left(-\frac{10}{96} \bar{\omega}_{1}\right)\right] \\
2896 \bar{\omega}_{1}=\bar{\rho} \frac{19600}{96^{2}} \bar{\omega}_{1} \\
\bar{\rho}=\frac{2896 \cdot 96^{2}}{19600} \cdot \frac{\bar{\omega}_{1}}{\bar{\omega}_{1}}=1361,71102 \\
\bar{\rho}=\frac{\mu \omega^{2} l^{4}}{2 \mathrm{EI}} \Rightarrow \omega^{2}=\frac{2 \mathrm{EI}}{\mu \mathrm{l}^{4}} \bar{\rho} \\
\omega^{2}=\frac{2 \cdot 2,1 \cdot 10^{11} \cdot 8,333 \cdot 10^{-7}}{18,055 \cdot 6^{4}} \cdot 1361,71102 \\
\omega^{2}=20367,2845 \\
\omega=142,714 \cdot \frac{1}{2 \pi}=22,725 \mathrm{~Hz}
\end{gathered}
$$

\subsection{The calculation method of FEM}

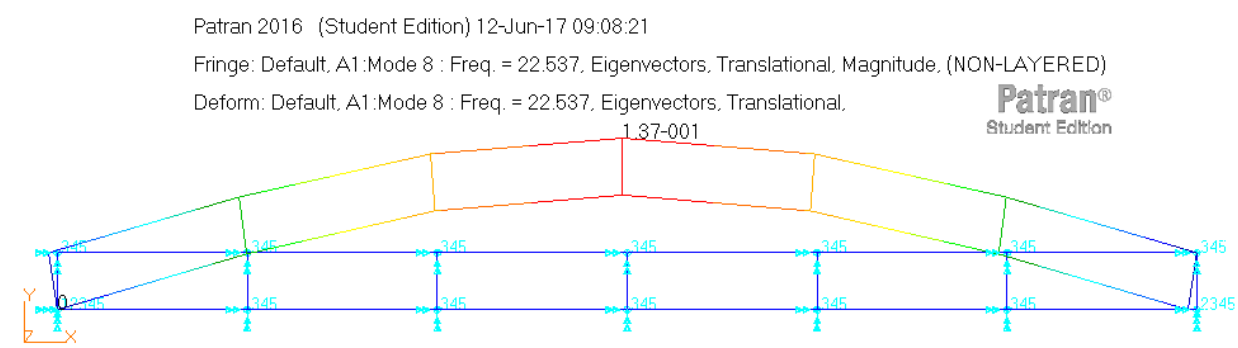

Fig. 8. First mode $(22,537 \mathrm{~Hz})$.

Table 2. Compare results.

\begin{tabular}{|c|c|c|c|}
\hline \multirow{2}{*}{$\begin{array}{c}\text { Circular } \\
\text { frequency }\end{array}$} & \multicolumn{3}{|c|}{ Calculation method } \\
\cline { 2 - 4 } & $\begin{array}{c}\text { Analytical solution } \\
\text { [Rzhanitsyn] }\end{array}$ & SAM & FEM \\
\hline $\begin{array}{c}\omega \\
\mathrm{rad} / \mathrm{s})\end{array}$ & 145,798 & 142,714 & 141,53 \\
\hline
\end{tabular}

\section{Conclusions}

The method of successive approximations (SAM) is used as a resolving method of differential equations.

The application of this method to the calculation of structures has a number of advantages:

- the ability to consider a simplified design scheme of the beam with a distributed load (instead of a system of concentrated forces);

- the ability to abandon the direct simulation of the design scheme in the design and computer complex (the time of which depends on the number of connections of the composite beam);

- gives the result of high accuracy at low time costs; 
The result is advisable to use at the initial stage of design as a preliminary, evaluation. In addition, it does not exclude the possibility of using the method as a test for structures, the calculation of which has already been made.

\section{References}

1. R. F. Gabbasov, Sali Musa, V. V. Filatov, V. S. Naumov, Estestvennye i tekhnicheskie nauki, 11, 656 (2015)

2. R. F. Gabbasov, V. V. Filatov, Chislennyj metod rascheta sostavnyh sterzhnej i plastin s absolyutno zhestkimi poperechnymi svyazyami (2014)

3. V. A. Smirnov, b. F. Kuzhin, iop conf. Series: earth and Envir., 90, 012200 (2017)

4. V. A. Smirnov, B. F. Kuzhin, Dni studencheskoy nauki, 416 (2016)

5. A. R. Rzhanitsyn, Compound rods (1986)

6. A. V. Dukart, A. I. Oleynik, Din. rasch. balok. (2002)

7. V. V. Filatov, Sborn. docl. PGS. MGSU, 177 (2004)

8. V. V. Filatov, VolgGASU, 2, 22 (2014)

9. V. A. Smirnov, Vibroprotection science equipment for high-precision measuring dissertation (2014)

10. S. Tudjono, Sunarto, A. L. Han, IOP Conf. Series: Mater. Sci. and Eng., 271, 012104 (2017)

11. G. Samadhan, Morkhade, M. G. Laxmikant, Steel and Comp. Struct., 23, 647 (2017)

12. A. M. Jamadar, P. D. Kumbhar, Intern. Res. J. of Eng. and Tech., 2, 2 (2015)

13. V. V. Filatov, Vestnik MGSU, 9, 23 (2013)

14. V. V. Filatov, N. A. Ryasniy, Teor. i prakt. rasch. zd., soor. i elem. konst.. An. i chisl. metody, 90 (2017)

15. M. I. Ganzhuntcev, V. V. Filatov, Izvestiya vysshih uchebnyh zavedenij. Tekhnologiya tekstil'noj promyshlennosti, 3, 203 (2017)

16. R. F. Gabbasov, V. V. Filatov, M. V. Aleksandrovskiy, Izvestiya vysshih uchebnyh zavedenij. Tekhnologiya tekstil'noj promyshlennosti, 3, 208 (2017)

17. B. G. Korenev, Spravochnik po din. soor. (1972)

18. G. M. Samadhan, M. G. Laxmikant, Intern. J. of Adv. Struct. Eng., 249 (2015)

19. M. R. Wakchaure, A. V. Sagade, Inter. J. of Eng. and Innov. Tech., 2, 1 (2012)

20. N. K. Chhapkhane, R. Kamble Shashikant, Inter. J. of Mech. Eng. App. Res., 3, 3 (2012) 